\title{
The pressure-amorphized state in zirconium tungstate: a precursor to decomposition
}

\author{
Akhilesh K Arora $^{1,3}$, V S Sastry ${ }^{1}$, P Ch Sahu ${ }^{1}$ and T A Mary ${ }^{2}$ \\ ${ }^{1}$ Materials Science Division, Indira Gandhi Centre for Atomic Research, \\ Kalpakkam 603 102, India \\ 2 Department of Materials Science, California Institute of Technology, Pasadena, CA 91125, USA \\ E-mail: aka@igcar.ernet.in
}

Received 12 August 2003, in final form 6 January 2004

Published 6 February 2004

Online at stacks.iop.org/JPhysCM/16/1025 (DOI: 10.1088/0953-8984/16/7/002)

\begin{abstract}
In contrast to widely accepted view that pressure-induced amorphization arises due to kinetic hindrance of equilibrium phase transitions, here we provide evidence that the metastable pressure-amorphized state in zirconium tungstate is a precursor to decomposition of the compound into a mixture of simple oxides. This is from the volume collapse $\Delta V$ across amorphization, which is obtained for the first time by measuring linear dimensions of irreversibly amorphized samples during their recovery to the original cubic phase upon isochronal annealing up to $1000 \mathrm{~K}$. The anomalously large $\Delta V$ of $25.7 \pm 1.2 \%$ being the same as that expected for the decomposition indicates that this amorphous state is probably a precursor to kinetically hindered decomposition. A $P-T$ diagram of the compound is also proposed.
\end{abstract}

\section{Introduction}

At high pressure many materials exhibit structural phase transitions, while some become amorphous [1-7]. Although it is widely believed that the phenomenon of pressure-induced amorphization (PIA) arises due to kinetic hindrance of equilibrium phase transitions, the structure of the equilibrium high-pressure phase, which the compound should have ideally evolved to, has remained speculative or unknown in most cases. Only in a few systems such as ice [1], quartz [2] and tetra-cyano-ethylene [8] can the high-pressure phase be identified from further measurements [9] and from computer simulations [10]. In this context, in addition to characterization of the $p$-amorphized state using x-ray diffraction and Raman spectroscopy, measurements of additional structural properties/features of this state such as its volume can be helpful in the identification of the equilibrium phase among different high-pressure polymorphs. The volume change across structural phase transitions is easily quantified from

3 Author to whom any correspondence should be addressed. 
the measurement of the lattice parameter. If the phase transition is irreversible, studies on the recovered samples can also yield this information. On the other hand, across amorphization a reliable estimate of volume change is difficult to obtain from x-ray diffraction due to the absence of sharp diffraction peaks in the disordered state. Earlier, a large-volume pistoncylinder apparatus was used for obtaining $\Delta V$ across amorphization in ice [1] and across the transformation of low-density a-SiO $\mathrm{S}_{2}$ to high-density a-SiO $\mathrm{S}_{2}$ [5]. Here we obtain the volume collapse $\Delta V$ across the irreversible pressure-induced amorphization in zirconium tungstate $\mathrm{ZrW}_{2} \mathrm{O}_{8}$, a material with large isotropic negative thermal expansion [11-14], from the linear dimension of the $p$-amorphized samples during isochronal annealing leading to the crystallization to the original cubic structure. The equilibrium state, which the system should have ideally evolved to, is identified from the magnitude of $\Delta V$. A $P-T$ diagram showing stable and metastable phases of the compound is also proposed.

\section{Experimental details}

In order to obtain the volume collapse the sample underwent during amorphization, we measured the longest dimension of the sample during cumulative isochronal annealing of $p$-amorphized zirconium tungstate platelets obtained by pressure soaking the samples to $5 \mathrm{GPa}$ for about $2 \mathrm{~h}$. Pressure treatment was carried out in a Bridgman opposed-anvil (diameter $30 \mathrm{~mm}$ ) high-pressure device consisting of a 500 ton hydraulic press with an electrically operated pump [15]. Granular sample powder, placed in a thin-walled (7.5 $\mu \mathrm{m})$ stainless-steel capsule of $5 \mathrm{~mm}$ diameter and $0.5 \mathrm{~mm}$ height, was mounted inside a pyrophyllite gasket, with steatite as the pressure-transmitting medium, and compressed to the desired pressure. Although amorphization is reported to occur at 2.2 GPa under hydrostatic conditions [14], we found incomplete amorphization at pressures lower than $5 \mathrm{GPa}$. In a single pressure treatment run, about $20 \mathrm{mg}$ of sample could be loaded. In most of the pressure treatment runs the recovered sample was in the form of a platelet with lateral dimensions ranging between 2 and $5 \mathrm{~mm}$ and thickness of about $0.3 \mathrm{~mm}$, while in some other runs the sample was fragmented into several small pieces. All annealings were carried out for $30 \mathrm{~min}$ in air. Dimension measurements were made at ambient temperature using an optical microscope of magnification $50 \times$ equipped with cross-wires and a calibrated $X-Y$ translation stage of least count $10 \mu \mathrm{m}$. This method of measurement of the change in size has the advantage that it avoids errors arising from the porosity, if any, of the as-synthesized samples.

\section{Results and discussion}

Annealing the samples at higher temperatures resulted in a substantial increase in their linear dimensions. Figure 1 shows the longest dimension of the samples, normalized to that after annealing at $1000 \mathrm{~K}$, as a function of annealing temperature. Note that a large increase in sample dimension takes place above $870 \mathrm{~K}$. In order to identify this state of the sample we recorded the $\mathrm{x}$-ray diffraction pattern after annealing the sample to $1000 \mathrm{~K}$. For recording the diffraction patterns the platelet sample was ground into fine powder and spread uniformly over a zerobackground sample holder made of $\langle 911\rangle$ cut silicon single crystal. Figure 2 shows the x-ray diffraction pattern of the annealed sample along with those of as-synthesized and $p$-amorphized samples obtained using $\mathrm{Cu} \mathrm{K} \alpha$ radiation. Note that there is a complete correspondence between the diffraction peaks of $1000 \mathrm{~K}$ annealed and the as-synthesized samples, indicating that the amorphous sample crystallized back to the original cubic phase upon annealing. Furthermore, the annealed sample does not show new/additional peaks. Note that the difference curve goes negative on either side of the centre of the strongest (210) peak. This is because of the slightly 


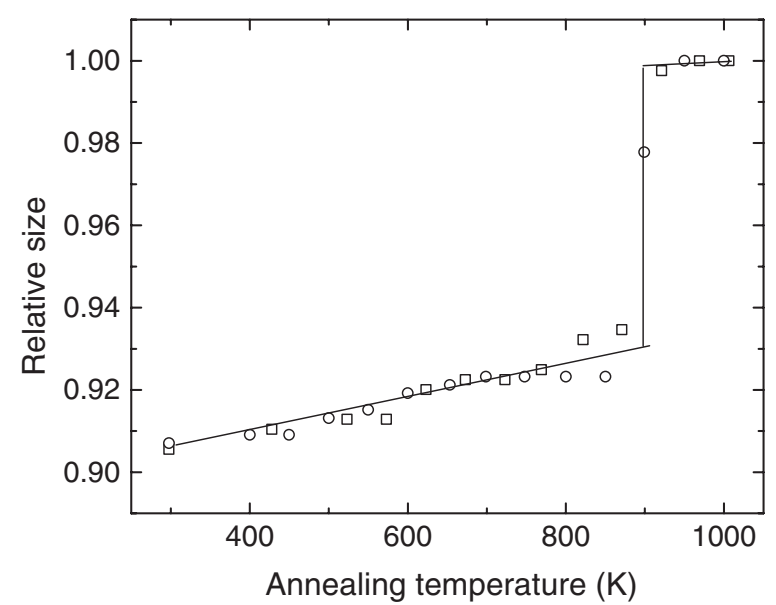

Figure 1. The longest linear dimension of $p$-amorphized $\mathrm{ZrW}_{2} \mathrm{O}_{8}$ samples after cumulative isochronal annealing to successively higher temperatures. The data presented here are from independent annealing experiments on separate samples and are normalized with respect to the sizes after annealing at $1000 \mathrm{~K}$. Sample A: O; sample B: $\square$. Note the large increase in sample length above $870 \mathrm{~K}$. The lines drawn through the points are guides to the eye.

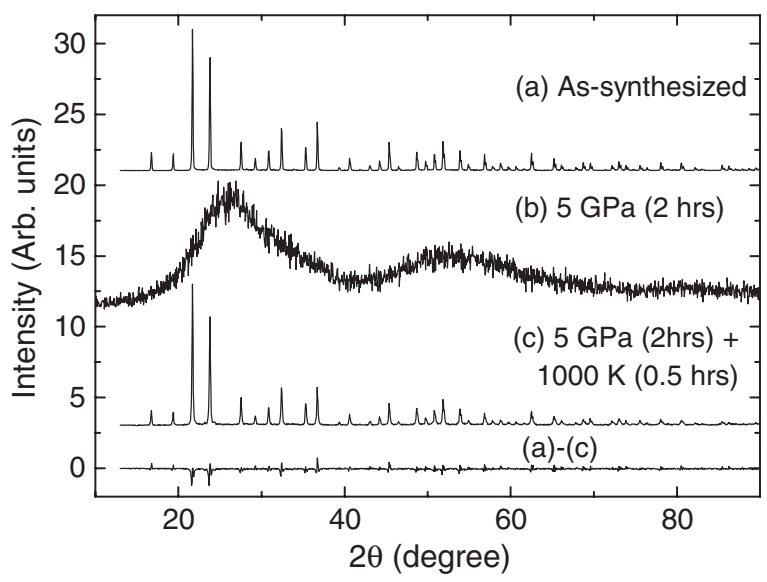

Figure 2. Powder $\mathrm{x}$-ray diffraction patterns of $\mathrm{ZrW}_{2} \mathrm{O}_{8}$ samples obtained using $\mathrm{Cu} \mathrm{K} \alpha$ radiation at ambient temperature and pressure after different pressure-temperature treatments. (a) Assynthesized, (b) after pressure soaking for $2 \mathrm{~h}$ at $5 \mathrm{GPa}$ and (c) after cumulative isochronal annealing of the $p$-treated sample to $1000 \mathrm{~K}$. The broad diffraction pattern in (b) arises due to amorphous nature of the $p$-treated sample. The diffraction peaks at large $2 \theta$ appear as a doublet due to the $\mathrm{K} \alpha_{1}$ and $\mathrm{K} \alpha_{2}$ wavelengths. The intensities of the patterns (a) and (c) are normalized with respect to those of the strongest (210) peak at $21.7^{\circ}$. For comparison, the (a)-(c) difference plot is also given.

larger width of the diffraction peak for the $1000 \mathrm{~K}$ annealed sample. The small difference in intensity of the other peaks, between the as-synthesized and the annealed samples, as seen in the difference plot, could be due to either a marginal positional disorder or a preferred orientation.

The annealed sample was further characterized using Raman spectroscopic measurements in the back-scattering geometry using the $488 \mathrm{~nm}$ line of an argon ion laser, using a set-up described elsewhere [16]. Figure 3 compares the Raman spectrum of the annealed sample with 


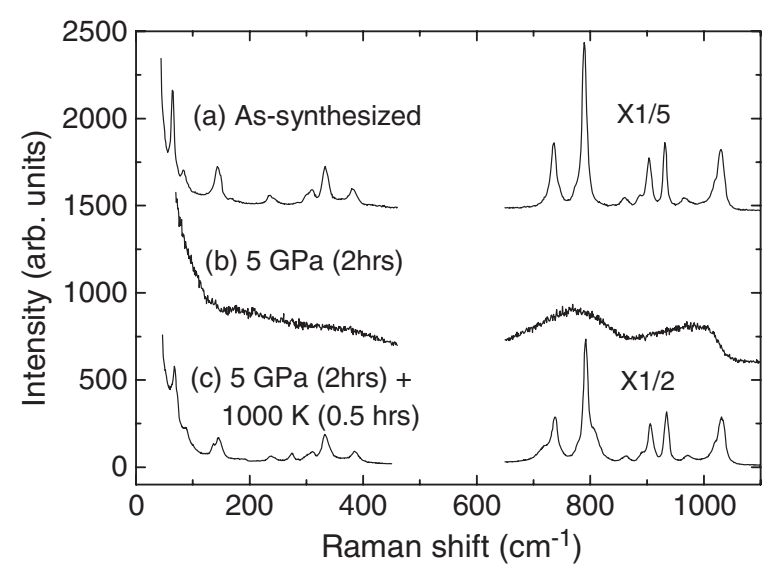

Figure 3. Raman spectra of $\mathrm{ZrW}_{2} \mathrm{O}_{8}$ samples at ambient temperature and pressure after different pressure-temperature treatments. (a) As-synthesized, (b) after pressure soaking for $2 \mathrm{~h}$ at $5 \mathrm{GPa}$ and (c) after cumulative isochronal annealing of the $p$-treated sample to $1000 \mathrm{~K}$. The broad bands in the spectrum (b) in the $v_{1}-v_{3}$ internal mode region of the tungstate ion arise due to the amorphous nature of the $p$-treated sample.

those of as-synthesized and $p$-amorphized samples. One can clearly see that the spectrum of the annealed sample has all the peaks of the as-synthesized $\mathrm{ZrW}_{2} \mathrm{O}_{8}$ confirming the recovery to the original cubic phase. However, in addition a weak peak at $272 \mathrm{~cm}^{-1}$ and a few weak shoulders at 136,712 and $810 \mathrm{~cm}^{-1}$ are found. As the x-ray diffraction pattern does not show any additional lines, the possibility of the new Raman features arising from other coexisting phase/phases can be ruled out. It may be mentioned here that an orientational disorder of polyatomic units/ions is known to result in the presence of more Raman lines than expected from group theory [17]. The structure of zirconium tungstate consists of interpenetrating sublattices of zirconium cations and tungstate anions, and its high-temperature phase has been reported to have oxygen disorder leading to two configurations of tungstate ions [18]. Hence the additional peaks may be attributed to the existence of some amount of residual orientational disorder of tungstate ions in the annealed samples. The disorder may also cause changes in the intensities of the diffraction peaks. These results thus suggest a nearly complete recovery to the original cubic phase upon annealing.

As the annealing at $1000 \mathrm{~K}$ resulted in recovery to the original cubic structure, one can estimate from the dimension of the annealed sample the extent of irreversible linear compression the sample had undergone during amorphization. We obtain this from the ratio of the size $L$ of the unannealed (amorphous) sample to that, $L_{0}$, after annealing to $1000 \mathrm{~K}$. From the data (figure 1) the linear compression $L / L_{0}$ turns out to be $0.906(5)$. Considering the amorphous phase to be isotropic, the volume compression $V / V_{0}$ then becomes $0.743(12)$, where $V$ and $V_{0}$ are the volumes in the amorphous and the cubic phases respectively. This implies that the irreversible volume collapse $\Delta V_{\mathrm{A}} / V_{0}=1-V / V_{0}$ upon amorphization relative to the cubic phase is as large as $25.7 \pm 1.2 \%$. A large change in the volume is also evident from the corresponding shift of the main peak of the amorphous sample to larger $2 \theta$ with respect to the two main peaks in the cubic phase. It may be mentioned that this material undergoes an intermediate cubic $(\alpha)$ to orthorhombic $(\gamma)$ transition at $0.3 \mathrm{GPa}$ prior to amorphization, which is also irreversible [19]. Among the known examples of high-pressure phase transitions, the volume collapse in the present case is anomalously large. Considering the present amorphous state to be metastable, we need to identify the final state, which this 
compound should have ideally evolved to, had the kinetics been adequate. In this context it is worth examining the present results in the light of a recent unified model of pressureinduced amorphization and decomposition [7]. According to this 'PIA could also occur if an equilibrium decomposition of the compound to more dense-packed daughter compounds is kinetically constrained'. An increase in temperature can provide sufficient kinetics for the decomposition to proceed. Several systems such as $\mathrm{Fe}_{2} \mathrm{SiO}_{4}, \mathrm{CuGeO}_{3}$ and $\mathrm{Mg}_{2} \mathrm{SiO}_{4}$ that are known to decompose under high-pressure-high-temperature conditions have been found to exhibit PIA at ambient temperature [3]. In view of the fact that $\mathrm{ZrW}_{2} \mathrm{O}_{8}$ can be synthesized from stoichiometric mixtures of $\mathrm{ZrO}_{2}$ and $\mathrm{WO}_{3}$ by sintering at high temperature, we consider the mixture of individual oxides as one of the possible decomposition routes. We calculate the volume change $\Delta V_{\mathrm{D}}$ upon decomposition of the compound according to the reaction $\mathrm{ZrW}_{2} \mathrm{O}_{8} \rightarrow \mathrm{ZrO}_{2}+2 \mathrm{WO}_{3}$ from the unit cell volume data of ambient pressure phases of the parent and the daughter compounds. Using $\Delta V_{\mathrm{D}}=V_{0}-\left(V_{1}+2 V_{2}\right)$, where $V_{1}$ and $V_{2}$ are the volumes per formula unit of the daughter compounds $\mathrm{ZrO}_{2}$ and $\mathrm{WO}_{3}$ respectively, we get $\Delta V_{\mathrm{D}} / V_{0}=0.267$. Note that $\Delta V_{\mathrm{A}}=\Delta V_{\mathrm{D}}$ within experimental error, i.e., the measured value of the volume collapse across the amorphization is the same as that expected for decomposition. It may be pointed out that $\Delta V_{\mathrm{A}} / V_{0}$ is quite different from that for the formation of a hexagonal phase of $\mathrm{ZrW}_{2} \mathrm{O}_{8}$ (reported fractional volume change $\sim 0.42$ [20]) under different $P-T$ conditions. This strongly suggests that the amorphous phase may be treated as a precursor to the decomposed state; however, one does not expect macroscopic growth of the individual daughter phases (disordered assemblage) to take place at ambient temperature. In view of this, one can view the amorphous phase as that of 'microscopically mixed' daughter compounds. One could in principle carry out a more rigorous calculation $[21,22]$ of $\Delta V$ as a function of pressure by taking into account the equations of state of the parent and the daughter compounds; however, as the volume change is very large in the present case, conclusions drawn from the ambient pressure data are also valid. The large magnitude of the volume collapse across amorphization in $\mathrm{ZrW}_{2} \mathrm{O}_{8}$ arises because its cubic phase can also be viewed as a loosely packed corner-linked network structure of $\mathrm{WO}_{4}$ tetrahedra and $\mathrm{ZrO}_{6}$ octahedra. On the other hand, the daughter compounds are very densely packed.

We now discuss a $P-T$ diagram of this technologically important, but metastable material. Zirconium tungstate is stable only between 1380 and $1505 \mathrm{~K}$ and it decomposes, if cooled slowly, into a mixture of constituent oxides. However, the metastable cubic phase can be quenched to the ambient and decompose only when heated above $1173 \mathrm{~K}$. In addition to the pressure-induced phase transition to the $\gamma-\mathrm{ZrW}_{2} \mathrm{O}_{8}$ phase [23] and subsequent amorphization [14], this compound also exhibits an order-disorder $(\alpha-\beta)$ transition at $428 \mathrm{~K}$ and ambient pressure [18]. Amores et al [24] have found, from x-ray diffraction measurements on recovered samples after high-pressure-high-temperature treatments, that it transforms to the $\gamma$-phase at $0.6 \mathrm{GPa}-873 \mathrm{~K}$, turns amorphous at $1 \mathrm{GPa}-873 \mathrm{~K}$ and decomposes into a mixtures of oxides at $0.6 \mathrm{GPa}-1073 \mathrm{~K}$. On the other hand, under different $P-T$ conditions, i.e., $1.4 \mathrm{GPa}-897 \mathrm{~K}$ and $2.2 \mathrm{GPa}-1042 \mathrm{~K}$, a hexagonal phase of $\mathrm{ZrW}_{2} \mathrm{O}_{8}$ has also been reported [20]. Thus the present results, along with those reported earlier, show the existence of three different paths of evolution of $p$-amorphized samples: (a) complete recovery to cubic structure upon annealing if pressure is released, (b) formation of hexagonal phase under high pressure and moderate temperatures and (c) decomposition of the compound at high pressure and relatively high temperatures. On the basis of these results as a function of pressure and temperature, we propose a $P-T$ diagram for $\mathrm{ZrW}_{2} \mathrm{O}_{8}$, in figure 4. The negative slope of the amorphization boundary is understandable because a moderate increase in temperature can increase the kinetics slightly and the onset of pressure-induced structural change (amorphization) can occur at a lower pressure. This has been found experimentally 


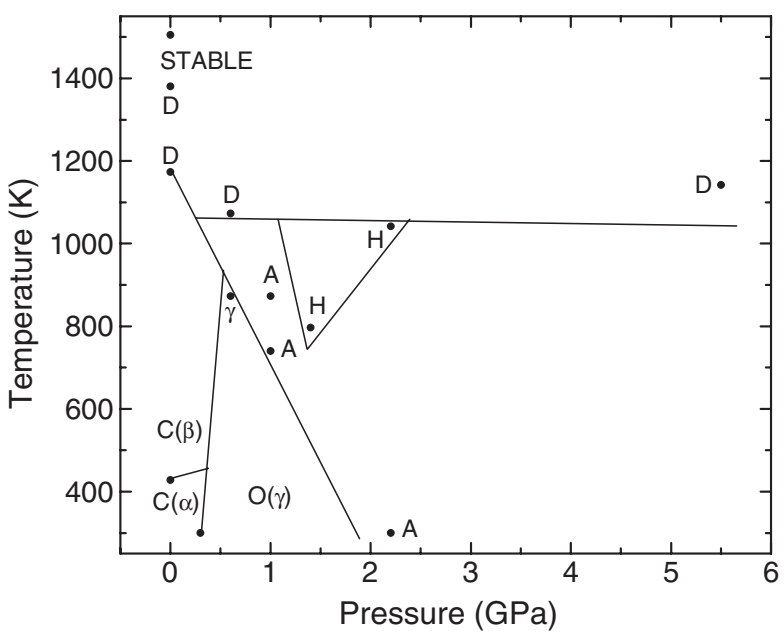

Figure 4. The proposed $P-T$ diagram of $\mathrm{ZrW}_{2} \mathrm{O}_{8}$. A: amorphous [14, 24]; D: decomposed state [20, 24]; $\mathrm{H}$ : hexagonal phase [20]; $\mathrm{C}(\alpha)$ : cubic $\alpha$-phase [11]; $\mathrm{C}(\beta)$ : cubic $\beta$-phase [18]; $\mathrm{O}(\gamma)$ : orthorhombic $\gamma$-phase [23]. The solid lines are the proposed phase boundaries.

also [20]. This $P-T$ diagram is not a phase diagram in the strict sense (a phase diagram is supposed to show only the equilibrium phases); however, it is useful as it identifies the regions of existence of different metastable phases from the point of view of applications. More measurements are required as a function of pressure and temperature to delineate the phase boundaries more precisely. Theoretical phase diagrams based on total energy calculations [25], not available for $\mathrm{ZrW}_{2} \mathrm{O}_{8}$ so far, are also desirable as these would throw further light on the phenomena of pressure-induced amorphization and decomposition in this system. Compounds that undergo irreversible amorphization have often exhibited polyamorphism [26], i.e., the existence of low- and high-density amorphous phases, such as those in quartz [5] and ice [27]. It would be interesting to investigate whether this compound also exhibits polyamorphism.

\section{Conclusions}

To conclude, the volume collapse across amorphization is obtained from the measurement of the linear dimensions of irreversibly $p$-amorphized samples of zirconium tungstate during their recovery to the original cubic phase upon isochronal annealing. The value of the volume collapse across the amorphization is found to agree very well with that expected for a decomposition of the compound. This strongly suggests that the final state, which the system would have ideally evolved to, is perhaps a mixture of $\mathrm{ZrO}_{2}$ and $\mathrm{WO}_{3}$. In view of this, the metastable $p$-amorphized state may be considered as a precursor to kinetically hindered decomposition rather than a phase transition. The present results also motivate the examination of other compounds and minerals that exhibit PIA from the alternative point of view of a possible decomposition.

\section{Acknowledgments}

It is a pleasure to acknowledge Professor Takehiko Yagi for stimulating discussions. We thank Dr T R Ravindran and Dr T Sakuntala for help in recording the Raman spectra, Dr B Viswanathan for keen interest in the work, Dr Baldev Raj for support and Mr S B Bhoje for encouragement. 


\section{References}

[1] Mishima O, Calvert L D and Whalley E 1984 Nature 310393

[2] Hemley R J, Jephcoat A P, Mao H K, Ming L C and Manghnani M H 1988 Nature 33452

[3] Arora A K 2002 High Pressure Phenomena (International School of Physics 'Enrico Fermi' vol 147) ed R J Hemley, G L Chiarotti, M Bernasconi and L Ulivi (Amsterdam: IOS Press) p 545 and references therein

[4] Richet P and Gillet P 1997 Eur. J. Mineral. 9907

[5] Mukharjee G D, Vaidya S N and Sugandhi V 2001 Phys. Rev. Lett. 87195501

[6] Ravindran T R, Arora A K and Mary T A 2001 J. Phys.: Condens. Matter 1311573

[7] Arora A K 2000 Solid State Commun. 115665

[8] Sahu P C et al 1989 Pramana-J. Phys. 33667

[9] Hemley R J, Chen L C and Mao H K 1989 Nature 338638

[10] Tse J S and Klug D D 1991 Phys. Rev. Lett. 673559

[11] Mary T A, Evans J S O, Vogt T and Sleight A W 1996 Science 27290

[12] Perrotoni C A and da Jornada J H A 1998 Science 280886

[13] Ernst G, Brohlom C, Kowach G R and Ramirez A P 1998 Nature 396147

[14] Ravindran T R, Arora A K and Mary T A 2000 Phys. Rev. Lett. 843879

[15] Sahu P C, Yousuf M and Rajan K G 1993 Physica B 183145

[16] Sakuntala T and Arora A K 2000 High Pressure Res. 171

[17] Sakuntala T, Arora A K, Shekar N V C and Sahu P C 1998 Europhys. Lett. 44728

[18] Evans J S O, Mary T A, Vogt T, Subramanian M A and Sleight A W 1996 Chem. Mater. 82809

[19] Evans J S O, Hu Z, Jorgensen J D, Argyriou D N, Short S and Sleight A W 1997 Science 27561

[20] Grzechnik A, Crichton W A, Syassen K, Adler P and Mezouar M 2001 Chem. Mater. 134255

[21] Fei Y and Mao H K 1993 J. Geophys. Res. 9811875

[22] Tschauner O, Mao H K and Hemley R J 2001 Phys. Rev. Lett. 87075701

[23] Jorgensen J D, Hu Z, Teslic S, Argyriou D N, Short S, Evans J S O and Slight A W 1999 Phys. Rev. B 59215

[24] Amores J M G, Amador U, Moran E and Franco M A A 2000 Int. J. Inorg. Mater. 2123

[25] Catti M, Fava F F, Zicovich C and Dovisi R 1999 Phys. Chem. Minerals 26389

[26] Poole P H, Grande T, Angell A and McMillan P F 1997 Science 275322

[27] Mishima O, Calvert L D and Whalley E 1985 Nature 31476 